\title{
Re-engineering Aerodynamics Education
}

\author{
David Darmofal \\ Associate Professor \\ Massachusetts Institute of Technology
}

\begin{abstract}
Aerodynamics curriculum in undergraduate engineering has reached a critical juncture forcing the need for change in the traditional, largely theoretical curriculum and lecturer/listener pedagogy. The role of aerodynamics in aerospace engineering, while still important, is no longer the dominant driver in aircraft design. Furthermore, industry, government, and academia --- the likely employers of aerospace graduates --- desire a workforce which is the more holistic and systems-thinking as opposed to the highly specialized, research-oriented engineer of past generations. Simultaneously, modern aerodynamics has been revolutionized by Computational Fluid Dynamics (CFD) while our undergraduate curriculum has largely neglected its use. Advances in the pedagogy of technical learning have also occurred which offer the potential to greatly improve the effectiveness of our teaching. In this paper, we report on a four year effort to re-engineer our aerodynamics education at the Massachusetts Institute of Technology. In particular, we discuss (1) the use of a semester-long aerodynamic design project to provide educational motivation and authentic learning experiences, (2) the application of active learning to improve student classroom engagement and student-faculty interaction, and (3) the integration of theoretical, experimental, and computational techniques into a modern aerodynamics curriculum. A variety of data are presented demonstrating improvements in student learning.
\end{abstract}




\section{Introduction}

In recent years, engineering curriculum reform has received serious attention from industry, government, and academic groups as the need for change in engineering education has become a well-recognized problem. Within aerodynamics, the need for re-engineering the traditional curriculum is critical. Industry, government, and (to some extent) academia has seen a significant shift away from engineering science and highly specialized research-oriented personnel toward product development and systems-thinking personnel. While technical expertise in aerodynamics is required, it plays a less critical role in the design of aircraft than in previous generations. In addition to these influences, aerodynamics has been revolutionized by the development and maturation of computational methods. At the same time, educational research in the sciences has demonstrated that learning can be significantly improved using pedagogical methods that differ from the standard lecture approach. These factors cast significant doubt that the traditional aerodynamics curriculum and pedagogy remain the most effective education for the next generation of aerospace engineers.

In this paper, we describe our four-year effort to reform our undergraduate aerodynamics education. The decision to strongly pursue educational reform in aerodynamics was stimulated not only by the external forces mentioned above but also by personal experiences teaching the subject. In particular, we had found that our students had very limited abilities to deal with aerodynamic problems that were different than the specific situations covered in the course. For example, the semester prior to modifying our curriculum, we developed a final exam to assess the students' ability (1) to apply concepts to different situations than encountered during the semester, and (2) to integrate concepts and apply them in a more complex, open-ended problem, i.e. the type of problems they would face as practicing engineers. The student performance on the exam was very poor and neither ability was demonstrated. Although we thought our students were achieving a deep level of conceptual understanding through our teaching, they were not. As a result, in the final exam, we assessed skills that the students did not have a good opportunity to develop through the subject's pedagogy. Since we felt strongly that conceptual understanding was a primary goal in our subject, we resolved to change our teaching.

In this paper, we will discuss three aspects of our reform work, specifically:

1. The use of a semester-long aerodynamic design project to provide educational motivation and authentic learning experiences.

2. The integration of theoretical, experimental, and computational techniques into a modern aerodynamics curriculum.

3. The application of active learning to enhance conceptual understanding.

The initial two years of this work was described by Darmofal et al (Darmofal et al 2001). This paper includes modifications we have made to refine the pedagogy since that initial report and, more importantly, includes a variety of data demonstrating the improved learning observed as a result of the new pedagogy. We begin the paper with a discussion of the course objectives. Then we discuss our use of project-based learning. In particular, we employ a semester-long design project that requires student teams to perform an aerodynamic design using models which they must first develop and validate. The design project, with its requirement for model development and validation, is the critical feature of our reformed pedagogy that allows effective integration of theory, experiment, and computation. Furthermore, the design project provides a broader context for learning the aerodynamics material that is the heart of the course. Next, we discuss features of our reformed pedagogy that target conceptual learning. Based on approaches developed in physics, we have developed a pedagogy that includes active learning in the classroom to address common misconceptions, pre-class homework to strengthen student preparation, and oral examinations to improve assessment of conceptual understanding. Finally, we present a variety of student performance and evaluation data that provides clear evidence of the benefits of the re-engineered curriculum.

\section{Course Objectives}

16.100 Aerodynamics is one of a set of upperclass subjects which undergraduates have the option of using to complete their degree requirements. The course is offered once a year in the fall semester and during the past four years the enrollment has been above 40 students (approximately $75 \%$ of our students take 16.100 during the undergraduate degree). Prior to this course, students have some exposure to basic fluid dynamics including conservation principles, potential flows, and some incompressible aerodynamics including thin airfoil theory and lifting line. 
As a first and important step in the re-engineering of this course, a set of course objectives were developed. These objectives are used to guide every aspect of the course. Specifically, students that successfully complete this course will be able to:

1. Formulate and apply appropriate aerodynamic models to predict the forces on and performance of realistic three-dimensional configurations;

2. Assess the applicability of aerodynamic models to predict the forces on and performance of realistic threedimensional configurations and estimate the errors resulting from their application;

3. Design and execute a computational and experimental aerodynamic analysis and design together with members of a team.

While the specific aerodynamic models could vary from semester-to-semester, in general, they will include: 2-D/ 3D potential flows (incompressible to supersonic) including panel and vortex lattice methods; boundary layer methods including the effects of transition and turbulence; coupled inviscid-viscous models; 2-D/3-D Euler and Navier-Stokes computations; wind tunnel testing.

For the first and second objectives, we specifically emphasize the prediction of not only aerodynamic forces but also aircraft performance. We believe that connecting the prediction of aerodynamic forces to aircraft performance (i.e. to the system performance) is critical to the development of engineering judgment in aerodynamics. Throughout the course, the students are often required to perform a sensitivity analysis to quantify how aircraft performance estimates (e.g. range, take-off distance, etc) are impacted by errors or uncertainty in aerodynamic predictions (e.g. drag coefficient, lift coefficient, etc). In addition, these objectives require the analysis of realistic three-dimensional configurations. The application of aerodynamics to reasonably complex configurations is an important learning experience as students must confront the approximations and uncertainties that are an inescapable part of all but the simplest engineering designs.

The third objective requires a team-based experience performing an aerodynamic analysis and design of a realistic three-dimensional configuration. The purpose of this experience is to expose the students to the respective roles of experiments, computations, and theory in a typical aerodynamic design cycle. The use of teams is largely a result of the intended complexity of the aerodynamic analysis and design that would be too difficult for a single individual to complete in the time given. However, when teams are working effectively, learning can be enhanced by team member interactions.

As the course objectives are difficult to assess directly, we follow an outcomes-based approach. Effectively, we assume that students demonstrating the abilities described by the measurable outcomes will have achieved the course objectives. These measurable objectives are given in the Appendix.

\section{Project-based Learning}

Modern aerodynamic design can be roughly broken into three phases: baseline concept generation, aerodynamic model development (including validation), and design trade studies using the validated aerodynamic models. Furthermore, modern aerodynamic design is really a blending of computational, experimental, and theoretical approaches where the strengths of all three are used to reduce uncertainty that the final design will meet its requirements. Typically, aerodynamics and other advanced engineering topics are taught with a significant focus on theory but little opportunity to apply theory especially to problems that approach the complexity faced in the design of modern aircraft. As a result, students perceive they are learning material 'just-in-case' they may need it later in their careers. In the proposed project-based approach, the knowledge is immediately being applied.

Educational literature has shown that project-based, more generally referred to as problem-based learning (PBL), has a wide variety of benefits. Interest in PBL arose in higher education in response to criticisms that programs in professional areas, e.g., medicine, engineering, failed to equip graduates with the problem-solving skills required for a lifetime of learning (Wilkerson \& Gijselaers, 1996). Problem-based learning has now become a widespread teaching method in disciplines, such as engineering, where students must learn to apply knowledge not just acquire it. Barrows (1996) describes the main features of PBL in this way:

- Learning is student centered, i.e., students make choices about how and what they want to learn.

- Learning occurs in small student groups and promotes collaborative learning.

- Teachers are facilitators or guides or coaches.

- Problems form the organizing focus and stimulus for learning. 
- Problems are a vehicle for the development of authentic problem-solving skills.

- New information is acquired through self-directed learning.

Not only is this style of learning more interesting and engaging for students, it also develops a greater understanding of the material since students find the information for themselves and then actively use the information and their skills to complete the project (Delisle, 1997).

Another important aspect of the design project is the opportunity to tie aerodynamic performance back to the system, i.e. the aircraft, and its performance. By focusing on the system-level impact of disciplinary design, students quickly learn to balance the perceived desire for accuracy in aerodynamic performance estimates with the actual need for accurate system performance estimates.

In summary, we feel that a well-designed design project can provide:

- Semester-long motivation and context for the learning of aerodynamics,

- Natural opportunities to integrate theoretical, experimental, and computational methods, and,

- Improved learning as has been described by a wealth of previous work in problem-based learning.

\section{Design Project Implementation}

Over the past four years, we have developed two design projects: one based on a military fighter aircraft and another on a blended-wing body commercial transport aircraft. In both cases, the projects utilized existing geometries for the baseline design that the student design teams then modified to improve some aspects of the vehicles performance. Prior to modifying the design, the students are required to develop and validate aerodynamic models to be used in the re-design of the existing baseline geometries. In particular, the validation process is critical to demonstrating how theory, experiments, and computations are combined in modern aerodynamic design.

A key feature of our design project implementation is weekly project work sessions. The goal of these project sessions is to provide a scheduled block of time in which the course staff (typically one faculty member and a teaching assistant) can interact with the teams as they begin to tackle the project. These two-hour sessions are held in a large electronic classroom with approximately 25 computers or roughly one computer for every two students. We have found that this ratio of computers-to-students is effective in promoting collaboration. Note, in addition to the two-hour project sessions, 16.100 has three hours per week of non-project class time. At the beginning of the semester, these project sessions are often used to provide information to the students about the project, clarify requirements, and introduce the various software tools and experimental facilities. However, later in the semester, the role of the staff tends more towards coaching and trouble-shooting.

The student teams consist of four-to-five students. Each team submits an interim and a final written report that is the basis for their grades. For the interim report, which is due roughly $2 / 3$ 's through the semester, the teams are required to fully describe all of the aerodynamic models they have developed including their validation studies. The final report focuses on using these validated models for design (in addition to correcting any errors found in the interim report). A best-practice which we have found for the design phase of the project is to require the teams to make a hypothesis on what design changes are likely to improve their ability to meet the design requirements based on their conceptual understanding of aerodynamic performance. Then, the final design phase becomes a study of whether the proposed design modifications have the desired effects; if not, the students are required to explain why their initial hypothesis was incorrect.

\section{Integration of Theory, Experiments, and Computation}

Our past aerodynamics curriculum had a significant focus on theoretical aerodynamics with some limited exposure to experimental and computational aerodynamics. However, as suggested by Murman \& Rizzi (2000), “today's aerodynamics engineer needs to be fluent in modern CFD methods and tools, and must know how to utilize them in conjunction with theory and experiment for aerodynamic analysis and design." While this seems an undeniable reality, the difficult problem is how best to integrate CFD into the mainstream aerospace curriculum.

We envision that the large majority of aerospace engineers will only have experience with the results of a CFD calculation, some engineers will be end-users of CFD, and a very small fraction will be involved in some aspect of CFD development. Thus, our general philosophy for integration of CFD into our undergraduate course was that the underlying aerodynamic approximations embodied by a computational tool must be well understood by a modern aerodynamicist, however, the details of the numerical methods are less important. Thus, we expect students to 
understand that a three-dimensional, compressible Euler calculation can model shock waves but, being inviscid, is not appropriate when viscous effects might be critical. Furthermore, we expect students to understand that a vortex lattice method, in addition to being inviscid, is a linear method that is only valid for thin bodies and small angles of attack. By contrast, we do not expect students to understand what a second-difference artificial dissipation operator is, or how a Roe approximate Riemann solver differs from Van Leer flux-vector splitting.

As can be observed from the measurable outcomes in the Appendix, a variety of computational methods are discussed including: 2-D panel methods, 2-D coupled integral boundary layer and panel methods, vortex lattice methods, and 3-D Euler and Navier-Stokes solvers. Furthermore, during the course of the semester (largely through the design project), the students actually apply all of these methods. We have found that the application of these methods can provide many important learning experiences. For example, in the fighter case study, students quickly learn that at low speeds, a vortex lattice method which runs in seconds is much more effective than a 3-D Euler solver which requires hours to complete. Or, when applying two-dimensional coupled boundary-panel methods to the airfoils of the fighter aircraft, the students experience first hand the sudden leading edge separation of thin airfoils at low angles of attack (and, almost as suddenly, realize the futility of using 2-D simulations for a low aspect ratio, highly swept wing).

We believe that all modern aerodynamicists should have a good grasp of the coupled but different roles of theory, experiment, and computation in the aircraft analysis and design. Thus, we devote a significant amount of class time to this issue, and, in fact, the project requires that the student teams develop validated aerodynamic models for all relevant operating conditions. In this process, students learn that neither CFD nor experiments are capable of providing reliable predictions for all applications, and understanding both the agreement or lack thereof between CFD and experiment is a crucial role for an aerodynamicist.

We also devote a portion of the class to wind tunnel testing. Perhaps more importantly, as part of the design projects, low speed wind tunnel tests are conducted in the M.I.T. Wright Brothers' Wind Tunnel. This low-speed tunnel, with its $7 \times 10$ foot cross-section, is very useful not only for the data which is produced but also for the handson experience students gain in flow visualization, transition and separation detection, wind tunnel models, etc. Furthermore, since the student teams are required to reduce and correct the raw wind tunnel data, they begin to appreciate how wind tunnel testing is as much of a model as purely theoretical or computational techniques.

\section{$\underline{\text { Lockheed Martin Fighter Aircraft Design Project }}$}

During the summer of 1999, we contacted several industry and government representatives requesting a design project that could serve as the semester-long theme of our aerodynamics course later that fall. Lockheed Martin Aeronautics Company (LMAC) proposed a project based on a typical re-design scenario encountered in the military aircraft industry. Specifically, the student teams were to develop aerodynamic models of an F-16-like wing-body geometry at several critical operating conditions and then use these aerodynamic models in a wing design trade study. The project was used for three semesters, Fall 1999, 2000, and 2001.

This project includes flight regimes from low subsonic to supersonic speeds including some at high angles of attack. The performance metrics of interest were:

1. The take-off distance at sea level conditions assuming the angle of attack is limited to a maximum of 25 degrees to avoid the tail striking the ground.

2. Radius of action (i.e. range) for Mach 0.9 cruise at $10 \mathrm{~K} \mathrm{ft}$

3. Dash time estimate from Mach 0.9 to Mach 1.2 at $10 \mathrm{~K} \mathrm{ft}$

For the subsonic (i.e. take-off) regime, a $1 / 9^{\text {th }}$-scale wind tunnel model was built and tested in the low-speed tunnel at M.I.T. At high speeds, experimental data was available from previous LMAC tests. The design phase of project focused on improving the take-off, cruise, and dash performance through introduction of leading and trailing-edge flaps, and variations in wing sweep and span.

\section{Boeing Blended-Wing Body Design Project}

For the Fall 2002 semester, a new design project was developed in collaboration with The Boeing Company based on the Boeing Blended-Wing Body aircraft design. The goal of this project was to redesign the baseline configuration to improve the static stability while minimizing drag and maintaining balance. Specifically, two flights conditions were considered: transonic cruise and low-speed approach. In approach, leading and trailing edge 


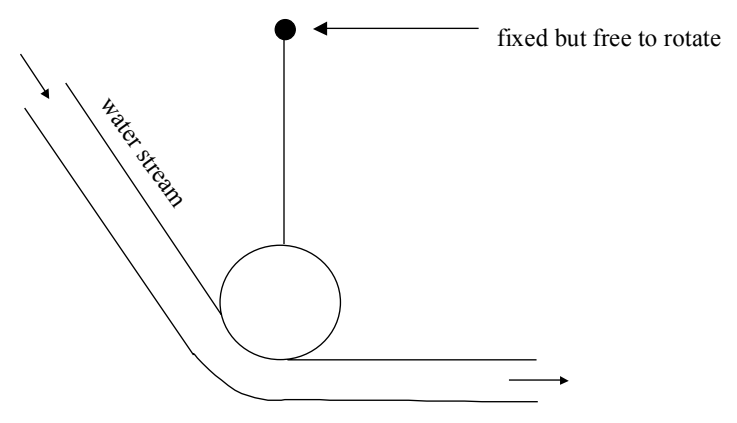

Given the water behaves as shown above, which direction will the cylinder rotate when the stream first
makes contact with the cylinder?
(a) Clockwise
(b) Counter-clockwise
(c) Not enough information

Figure 1: Flow turning and momentum change concept question

devices were permitted to be active, while in cruise, the aircraft was required to be clean. As in the fighter aircraft project, low speed wind tunnel tests were performed to provide validation data for the aerodynamic models.

\section{Active Learning and Assessment}

One strategy for strengthening conceptual learning is a set of pedagogical methods known as active learning. Unlike traditional lectures, active learning seeks to engage the students with the material in class. When implemented properly, active learning can clarify common misconceptions, improve higher order thinking, encourage self-driven learning, decrease feedback time between faculty and students, etc (Crouch \& Mazur, in press; Felder \& Brent, 1996; Hake, 1998; Heller \& Hollobaugh, 1992; Heller, Keith, \& Anderson, 1992; Johnson \& Johnson, 1989, 1996; and Meltzer \& Manivannan, 1996). However, most uses of active learning have been carried out in science education at the elementary, secondary, and undergraduate levels, while only a few applications (Mourtos, 1997) have been made to engineering education.

During the Fall 1999 semester, we began using a peer instruction approach, similar to that developed for physics by Mazur (1997). In this approach, conceptual questions (referred to as ConcepTests by Mazur) are given to students in class with time for individual thought and reflection. After a check to see how well students have understood a question, small group discussions may be held. In addition, the instructor will usually clarify misconceptions and lead students in further exploration of the concept often giving a mini-lecture. In a typical class, two-to-three concept questions are usually discussed. Several options exist for measuring the class understanding. In 16.100, we have found the use of a handheld Personal Response System (PRS) to be very effective. PRS has several advantages over hand-raising or flash cards including anonymity of student responses and the efficient generation of assessment data to analyze aggregate performance.

To illustrate a typical concept question, we will consider the generation of lift. The generation of lift on an airfoil is filled with many misconceptions due to the (usually inaccurate) folklore regarding how airplanes fly and further complicated by the knowledge gained in previous courses. We use a series of concept questions concentrating on understanding lift generation through momentum changes, streamline curvature, and reaction forces. The first question involves the impingement of a water jet on a cylinder as shown in Figure 1. Although many students believe the jet will cause the cylinder to be propelled away from the stream, in actuality, the object will rotate into the stream. A simple momentum balance leads directly to the connection between force (lift) generation and momentum change --- our intended result! When we use this question, we include an in-class demonstration that clearly demonstrates the cylinder being drawn into the stream. This question is then followed by a series of questions connecting the concept of flow turning to force generation, and extending the ideas to understand the loss of lift at stall when the airfoil no longer turns the flow as effectively.

Our experience with concept questions has shown that the students must have some experience with the material prior to class (see also Mazur for a discussion of the need for student preparation). Otherwise, discussing concepts and misconceptions is nearly impossible since students are not likely to have encountered much of the material prior 


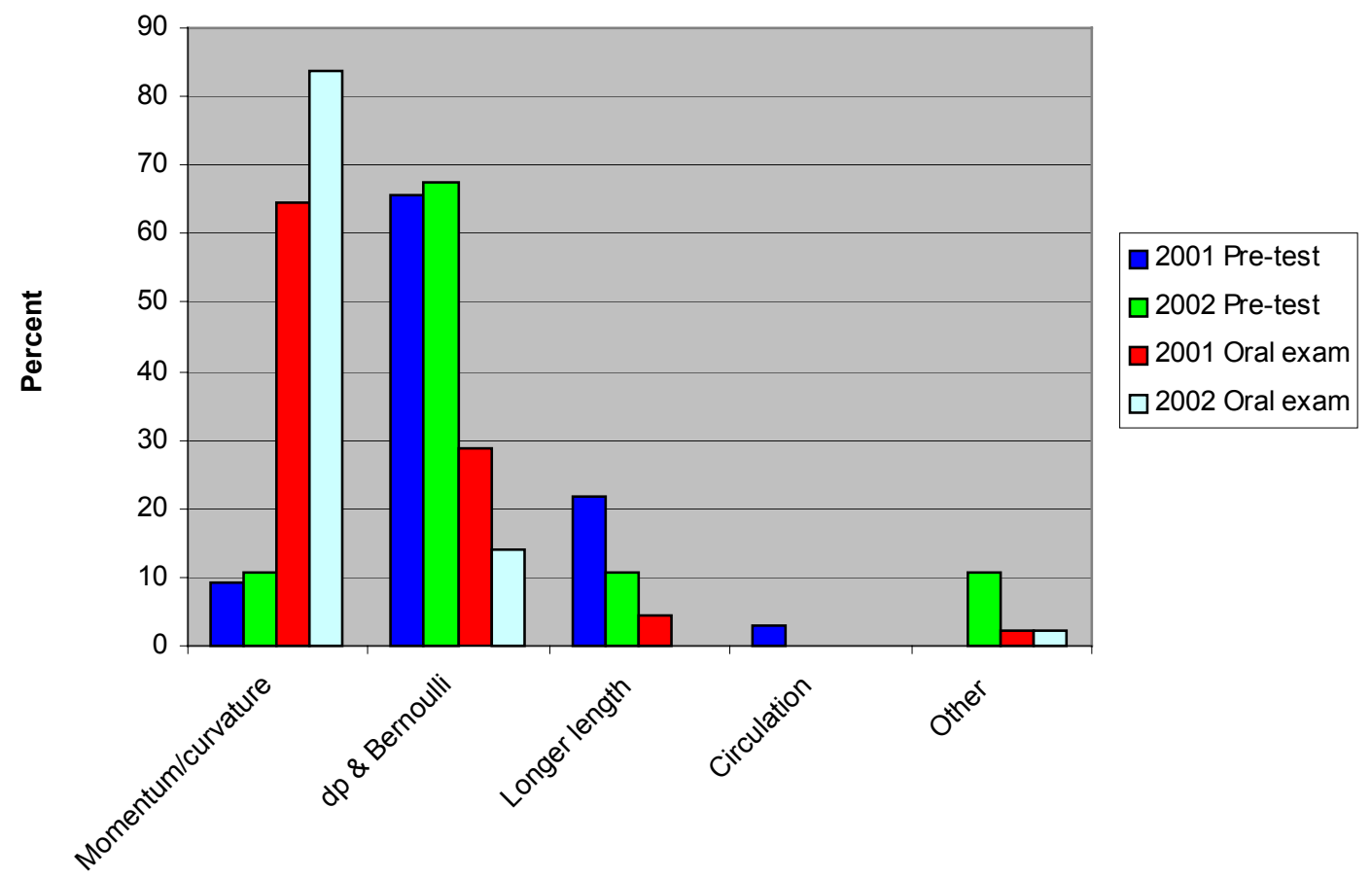

Figure 2: Changes in conceptual understanding of the generation of lift on an airfoil during Fall 2001 and Fall 2002 semesters.

to the course. In our aerodynamics course, we give reading assignments and graded homework that are due prior to discussing the material in class. The use of pre-class homework is a significant shift from traditional engineering pedagogy in which homework is assigned and due only after discussing the material in class. Not only is the preclass homework critical to the success of active learning in the classroom but it also encourages student selflearning. Furthermore, by scanning the homework assignments, student misconceptions and common difficulties can be detected immediately rather than only week(s) after discussing material. By encouraging self-directed learning through pre-class homework, students are better prepared for class and faculty can then focus on the important concepts and misconceptions.

In addition to changing our in-class pedagogy, we have also modified our exams from a written to an oral format. While written exams can only analyze the information which appears on paper, i.e. the final outputs of a student's thought process, an oral exam is an active assessment which can provide greater insight into how students understand and relate concepts. Also, oral exams are adaptive to each student. If a student is stuck or has misunderstood a question, the faculty can help the individual. As opposed to a wasted assessment opportunity, the dynamic adaptivity of an oral exam raises the likelihood of an effective assessment. Finally, practicing engineers are faced daily with the real-time need to apply rational arguments based on fundamental principles. By using oral exams, we can directly assess this ability.

\section{Results}

Quantifying the impact of pedagogical change on learning is difficult. Our approach is to take data from a variety of sources and draw our conclusions from the aggregate. While any single source is suspect, taken together, the results become convincing.

\section{Conceptual Understanding: Generation of Lift}

We begin by looking at whether student conceptual understanding has improved for specific concepts that were targeted during the semester. For the past two years, data has been gathered on the students' conceptual understanding of lift generation on an airfoil. In a pre-test at the beginning of the semester, students were asked to explain the generation of lift qualitatively. Then, this understanding was tested in later exams (in Fall 2001 it was 


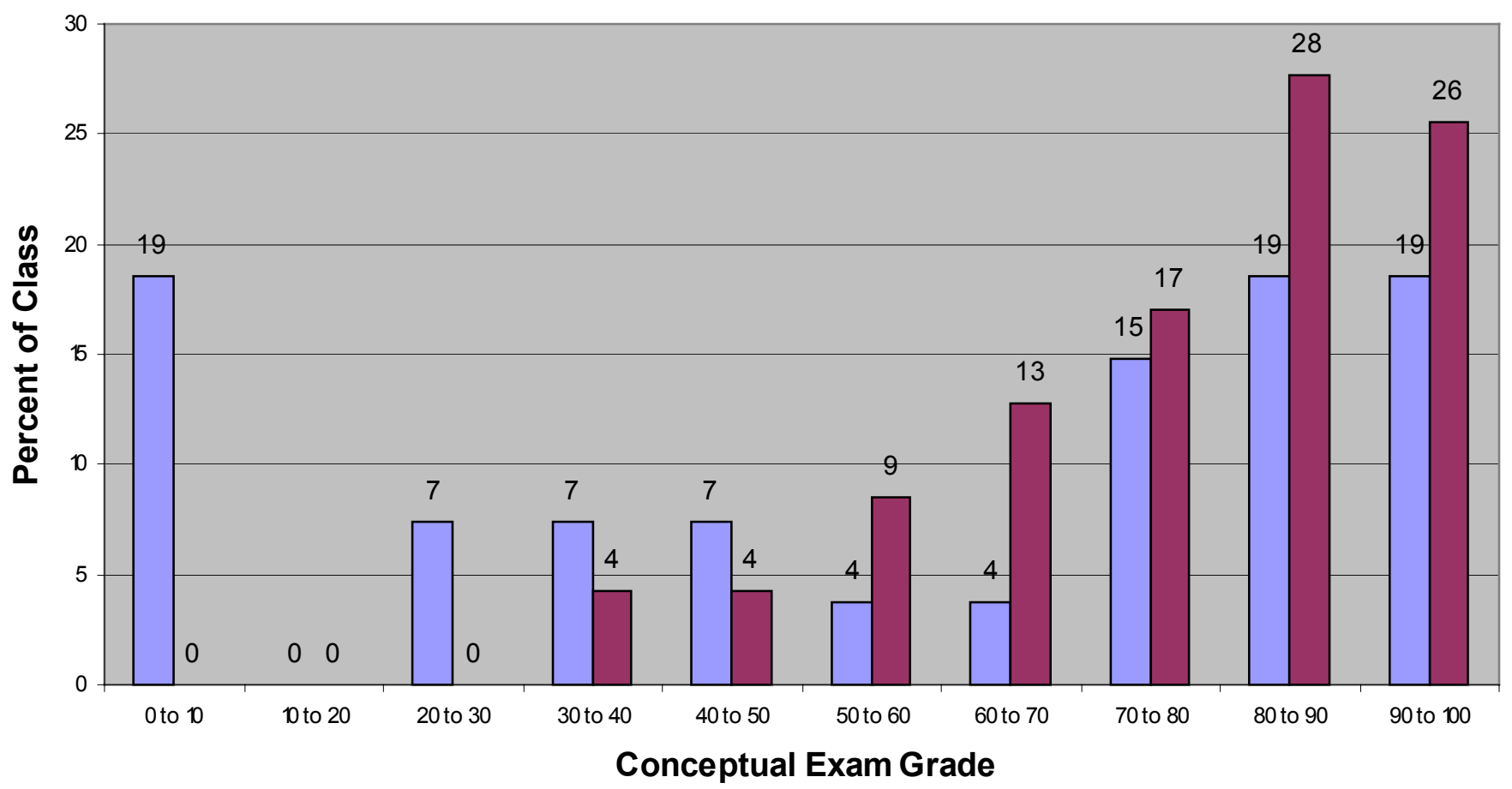

Figure 3: Comparison of student performance on the same conceptual question from 2000 written exam (blue) and 2001 oral exam (maroon).

tested in the final oral exam and in Fall 2002 it was tested in the mid-term oral exam). Figure 2 illustrates how the students' understanding of lift has changed. As explained above, we stress the understanding of lift generation through momentum changes and streamline curvature. At the beginning of the semester, only $10 \%$ of the students offer this explanation. For the second response, students correctly explain that a net pressure difference is acting on the airfoil to produce lift, but then offer the Bernoulli effect as the underlying cause of this pressure difference. This often leads to the frequently-cited (but incorrect) explanation that the larger length on the upper surface is responsible for the larger velocities and lower pressures compared to the lower surface. At the beginning of the semester, this is the most popular answer at over $60 \%$. However, by the exam assessments, the large majority of students properly explain lift generation through flow turning and momentum changes. A handful of other explanations are offered as well, though in much smaller numbers.

\section{$\underline{\text { Synthesis of Concepts }}$}

We have also assessed the students' ability to integrate several concepts using a question from the Fall 1998 final written exam as the basis for the 2001 final oral exam. Note, the Fall 1998 final exam was the exam just prior to reforming the aerodynamics curriculum. The question asked the students to propose an aerodynamic model for the refueling boom of a tanker. The refueling boom was assumed to have a cylindrical cross-section and was oriented at an angle to the subsonic freestream. Also, a high aspect ratio wing was mounted at the end of the boom for position control. Specifically, the students needed to:

- Explain the dominant sources of drag on the boom

- Account for the possibility of compressibility effects and recognize that the angled boom was equivalent to a swept wing in terms of the critical Mach number

- Explain and estimate the lift and drag (induced and skin friction) on the high aspect ratio control wing

- Account for the effects of downwash due to the tanker's wing

As shown in Figure 3, a significant improvement in performance has been observed. For example, 16\% more of the students scored in the $80-100 \%$ range during the Fall 2001 semester. However, several caveats exist. In particular, in the written exam, students had several other questions to answer and could adopt the strategy of spending less time on this specific question. Thus, we believe that the apparent performance gains were in part due to the more effective assessment strategy and in part due to stronger conceptual understanding. 


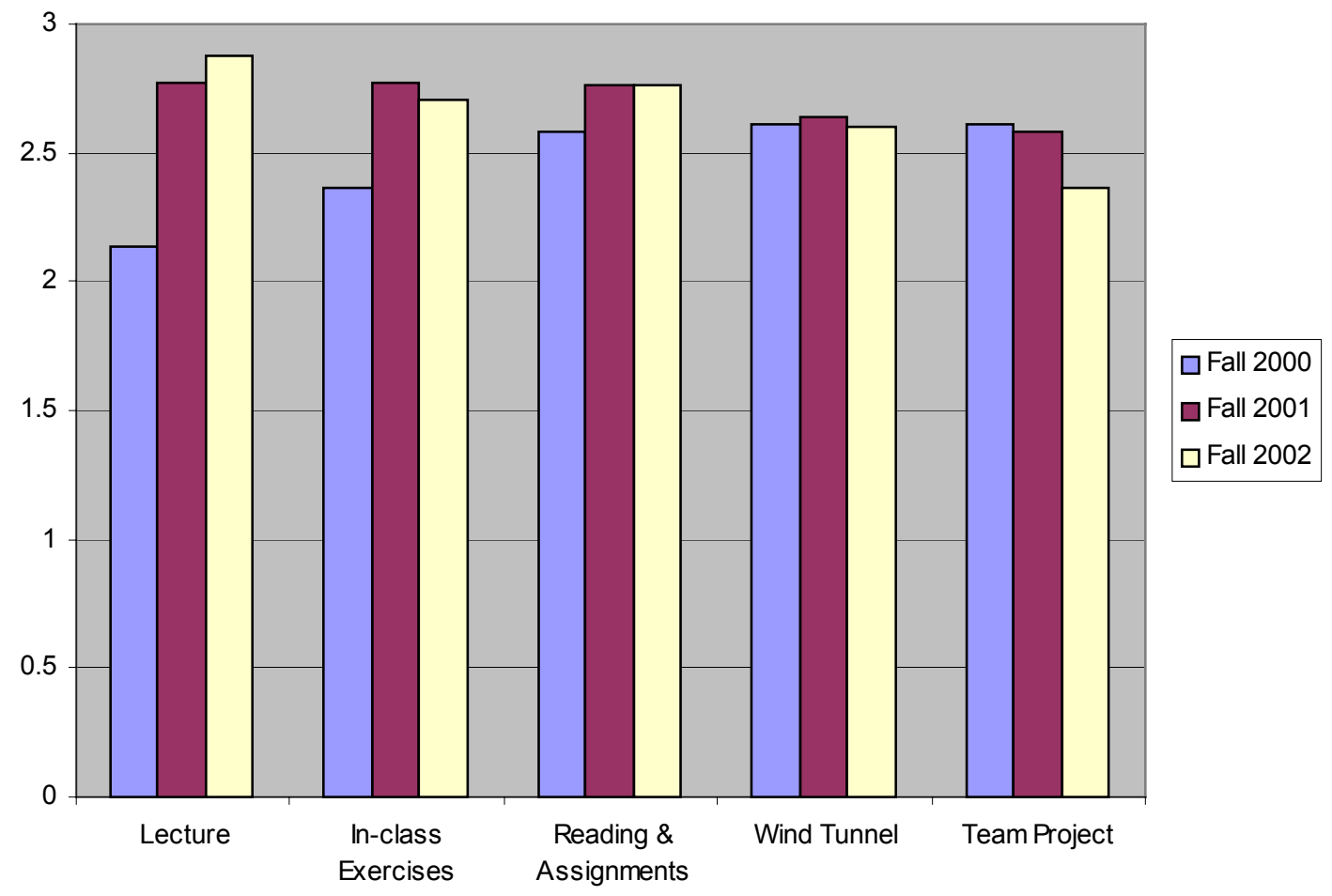

Figure 4: Student ratings of the effectiveness of different aspects of the course pedagogy. $1=$ not effective, $2=$ effective, and $3=$ very effective

\section{$\underline{\text { Impact of Implementation }}$}

While student reactions to the new pedagogy have been overwhelmingly positive in the past two years, they have varied from year-to-year. In particular, during the process of reforming the curriculum, the implementation of the pedagogy was improved with each year's experience. In Figure 4, end-of-semester student evaluations from Fall 2001 clearly show a dramatic improvement in effectiveness over Fall 2000 for the lectures, in-class exercises, and assignments. We note that in both years, we used active learning but in Fall 2000, our pre-class assignments were not difficult and required little student engagement of the material to answer. As a result, the students were not as well prepared for in-class active learning and the students found the overall approach to be less effective. We also note that the team project effectiveness dropped in the Fall 2002 semester; we believe this to be linked to the introduction of the new project based on the blended wing body. As this new project had problems that were solved during this first offering, we expect that the ratings for the project will improve in coming years.

\section{$\underline{\text { Student Comments on Pedagogy }}$}

Student evaluation comments describe a consistent view with respect to learning improvement as observed in the previous performance data (and staff observations). We briefly mention some specific comments on the various aspects of the pedagogy. Some typical student responses with respect to the design project include:

- I think the team projects are really good. There are some kinks which need to be worked out and possibly explained sooner, but they really bring us to an understanding of what elements are necessary to incorporate theory into design.

- $\quad$ The projects were very interesting. Learning how to use computational tools and seeing how all the theory and testing is used in conjunction to gain accurate results was very useful and enjoyable.

- In a project, you have to take what you learn and directly apply to something. This is more effective than a problem set because it is on a larger scale - while on a problem set you may only perform a calculation once, a 
project makes you do that many more times. You begin to understand why and when what you are doing is applicable on a much deeper, intimate level.

- I think I learned the must during the project reports, since we really had the chance to experience the empirical approach in the wind tunnel, as well as its complementary part doing regular research. The learning experience was particularly enriching, since we got to share our knowledge with the team and thus get over certain gaps in the subject.

The students have perceived the educational benefits of applying the material they are learning in class on a complex problem; furthermore, several students (including other comments not shown here) note that the project allowed them to better appreciate how theory and computation complement experiments in aerodynamic design. The only consistent negative comment on the design project over all of the four years is one of implementation. Students from all of these years comment that the project seems overwhelming and unorganized at the beginning of the semester. Though some of this lack of organization is unfortunately accurate (especially during the first year of using a new project as described above), some of the lack of structure is done purposely to require the students to develop their own approaches to meeting the project requirements.

The evaluation comments on the pre-class homework and active learning are particularly remarkable for the uniformly favorable nature. For example:

- Doing homework before the lectures is good... makes actual learning in lectures possible.

- Prof. Darmofal forces you to learn the subject material by assigning homework that he has not covered in lecture, therefore I have to force myself to read the text and go to office hours. When he does go over in lecture after the Pset is due, I did absorb the material much better.

- The teaching methods are outstanding. Prof. Darmofal is great at teaching the material and making us read before the p-set is good form.

- I was initially opposed to the idea that I had to do reading \& homework before we ever covered the subjects. Once I transitioned I realized that it made learning so much easier!!

- I was skeptical at first of new techniques like PRS, hw on material that hasn't been learned in lecture. In the end, it worked out very well. This has been a course where I really felt like I got my money's worth.

While students often mention an initial opposition to the strategy, they demonstrate a transition in their learning style as they recognize the effectiveness of the approach.

Similar reactions are observed with respect to the oral exams:

- $\quad$ The oral exam was a different learning assessment approach that I liked a lot.

- I really like oral exams that stress conceptual knowledge.

- The oral exams are an excellent measure of understanding.

- Oral exams [are the best part of the subject], I think these gave a good opportunity to show what you understand.

- Oral exams are also good. Pretty nerveracking, but good overall.

Again, these excerpts are representative of the great majority of comments on the effectiveness of oral exam assessments. Surprisingly, though most students have never had an oral exam prior to this course, they find the process to be a much more accurate representation of their understanding. As a passing note, we mention that since the initial successful use of oral exams in the aerodynamics course, they have now been adopted by several other undergraduate (and some graduate) courses in the department.

\section{Conclusions}

In response to external and internal forces of reform, we have re-engineered our undergraduate aerodynamics curriculum. While the reform can involve substantial effort, the results as measured by student performance and student evaluation clearly demonstrate that significant improvements in learning can be achieved. Furthermore, the introduction of a semester-long team project that embeds the students in a realistic design cycle offers the opportunity to address the role of experiment, theory, and computation in modern aerodynamic design. In this age where the breadth of aerospace engineering curriculum is being expanded and traditional disciplines have become a smaller portion of an overall aerospace undergraduate program, these advances in pedagogical effectiveness are critical to the adequate education of the next-generation of aerospace engineers. 


\section{Acknowledgements}

This work over the past four years has involved significant interactions both internal and external to MIT. The initial re-design of the curriculum began as a collaboration with Prof. Earll Murman. The original case study was developed with Lockheed Martin by Mike Love and Dennis Finley. The new case study based on Boeing's Blended Wing Body was developed with Bob Liebeck (a Boeing employee and a Professor of the Practice at MIT). During the Fall 2000 semester, 16.100 was co-taught with Professor Steve Ruffin who was on sabbatical from Georgia Tech. This work was partially supported by a National Science Foundation CAREER and a MIT Alumni Fund grant for Educational Innovation.

\section{References}

Anderson, J.D. (1991) Fundamentals of Aerodynamics, Second Edition, McGraw Hill.

Barrows, H. S. (1996). Problem-based learning in medicine and beyond: A brief overview. In Wilkerson, L., \& Gijselaers, W. H. (Eds.) Bringing problem-based learning to higher education: Theory and practice. New Directions for Teaching and Learning, No. 68. San Francisco, CA: Jossey-Bass.

Crouch, C. H., \& Mazur, E. (in press). Peer instruction: Eight years of experience and results. (Available http://mazur-www.harvard.edu).

Darmofal, D.L., Murman, E.M., \& Love, M. Re-engineering Aerodynamics Education. AIAA Aerospace Sciences Meeting, AIAA Paper 2001-0870, Reno, NV 2001.

Delisle, R. (1997). How to use problem-based learning in the classroom. Alexandria, VA: Association for Supervision and Curriculum Development.

Felder, R. M., \& Brent, R. (1996). Navigating the bumpy road to student-centered instruction. College Teaching, 44, $43-47$.

Hake, R. R. (1998). Interactive-engagement vs. traditional methods: A six-thousand-student survey of mechanics test data for introducing physics courses. American Journal of Physics, 66 (1), 64-74.

Heller, P., \& Hollobaugh, M. (1992). Teaching problem solving through cooperative grouping. Part 2: Designing problems and structuring groups. American Journal of Physics, 60 (7), 637-644.

Heller, P., Keith, R., \& Anderson, S. (1992). Teaching problem solving through cooperative grouping. Part 1: Group versus individual problem solving. American Journal of Physics, 60 (7), 627-636.

Johnson, D. W., \& Johnson, R. T. (1989). Cooperation and competition: Theory and research. Edina, MN: Interaction Book Company.

Johnson, D. W., \& Johnson, R. T. (1996). Meaningful and manageable assessment through cooperative learning. Edina, MN: Interaction Book Company.

Mazur, E. (1997). Peer instruction: A user's manual. Upper Saddle River, NJ: Prentice Hall.

Meltzer, D. E., \& Manivannan, K. (1996). Promoting interactivity in physics lecture classes. Physics Teacher, 34, 72-76.

Mourtos, N. J. (1997). The nuts and bolts of cooperative learning in engineering. Journal of Engineering Education, 86 (1), 35-37.

Murman, E.M. \& Rizzi, A. Integration of CFD into aerodynamics education. Frontiers of Computational Fluid Dynamics - 2000. Editors: D.A. Caughey and M.M. Hafez. 2000.

Wilkerson, L. \& Gijselaers, W. H. (Eds.). Bringing problem-based learning to higher education: Theory and practice. New Directions for Teaching and Learning, No. 68. San Francisco, CA: Jossey-Bass 


\section{Appendix: Measurable Outcomes}

As the course objectives are difficult to assess directly, we follow an outcomes-based approach. Effectively, we assume that students demonstrating the abilities described by the measurable outcomes will have achieved the course objectives. The outcomes are listed below including the assessment methods used for each. Specifically, students successfully completing 16.100 will be able to:

1. Apply flow similarity, non-dimensional coefficients such as the lift and drag coefficient, and non-dimensional parameters such as the Mach number and Reynolds number in aerodynamic modeling of realistic configurations (homework, team project reports, exams)

2. Apply integral momentum conservation to explain the relationship between flow turning, the generation of lift on an airfoil, and the subsequent loss of lift upon stall (homework, exams)

3. Explain the sources of friction, induced, wave, and pressure drag (homework, exams)

4. Explain the motion and deformation of a fluid element using kinematics including the definition of shear strain, normal strain, vorticity, divergence, and the substantial derivative (homework, exams)

5. (a) Explain the concept of a laminar boundary layer including the definition of the displacement thickness, the momentum thickness, and the skin friction coefficient, and the importance of the Reynolds number in determining the presence and behavior of a boundary layer (homework, exams), and (b) Apply the integral boundary layer equations to describe the qualitative evolution of a laminar boundary layer including separation and to quantitatively estimate the local thickness and skin friction (homework, exams)

6. Explain the onset of turbulence in a boundary layer (i.e. transition) and the qualitative effects of turbulence on boundary layer evolution including the impact on velocity profile, skin friction coefficient, boundary layer thickness, and separation (homework, exams)

7. Estimate friction drag on 2-D and 3-D configurations by decomposing the geometry into patches and assuming appropriate local values of skin friction coefficients including the possibility of laminar or turbulent boundary layer conditions (homework, team project reports, exams)

8. Explain the basic elements (see Comment on basic elements below) of 2-D panel methods and 3-D vortex lattice methods (homework, exams)

9. Explain the basic elements of coupled inviscid-viscous models for 2-D airfoils (homework, exams)

10. (a) Explain the basic elements of thin airfoil potential flow models for 2-D subsonic and supersonic flows (homework, exams), and (b) Apply thin airfoil potential flow models to estimate the forces on airfoils in 2-D subsonic and supersonic flows (homework)

11. (a) Explain the basic elements of the lifting line model for high aspect ratio wings (homework, exams), (b) Describe the dependence of lift and induced drag on geometry and performance parameters (e.g. aspect ratio, twist, camber distribution, wing loading, flight speed, etc) using the lifting line model (homework, exams), and (c) Apply the lifting line model to estimate lift, induced drag, and roll moments on high aspect ratio wings (homework)

12. Explain the basic elements of the finite volume approximation to the compressible Euler and Navier-Stokes equations (homework, exams)

13. (a) Explain the relationship between sound propagation and shock waves (exams), (b) Describe the qualitative change in flow conditions (Mach number, pressure, temperature, total pressure, etc.) across shocks and expansion fans (exams), (c) Estimate the change in flow conditions across shocks and expansion fans using shock-expansion theory (homework) (d) explain transonic drag rise including the critical Mach number and the use of wing sweep to delay drag rise (homework, exams). 
14. Explain the use of wind tunnel testing in aerodynamic modeling focusing on the importance of flow similarity in scale testing and on the typical corrections (e.g. wall corrections) required to simulate flight conditions (homework, team project reports, exams)

15. Assess the ability and limitations of an aerodynamic model to estimate lift and drag (separated into friction, induced, wave, and pressure drag contributions) for a specific application (homework, team project reports, exams)

16. Apply linear and non-linear sensitivity analysis to quantify the impact of error or uncertainty in aerodynamic predictions on the prediction of flight vehicle performance (homework, team project reports)

17. Contribute substantially as an individual to the design and execution of a computational and experimental aerodynamic analysis of realistic 3-D configuration together with members of a team (team project reports) 